\title{
ATYPICAL PRESENTATION OF LIMITED CUTANEOS SYSTEMIC SCLEROSIS
}

Rita Marina Soares de Castro Duarte ${ }^{1, *}$, Caroline Diniz Bezerra ${ }^{1}$, Leonardo Fernandes e Santana ${ }^{1}$, Mateus de Sousa Rodrigues ${ }^{1}$, Luana Barros Caxias de Souza ${ }^{1}$

1.Universidade Federal do Vale do São Francisco, Petrolina (PE), Brazil.

*Corresponding author: ritinhaww@hotmail.com

\section{BACKGROUND}

Systemic sclerosis (SSc) is a collagen disease characterized by inflammatory and fibrotic changes in multiple organs, including the heart, lungs, kidneys, gastrointestinal tract, blood vessels and skin. Its broad spectrum of clinical presentation may mimic several systemic diseases. This case describes an atypical initial presentation of limited cutaneous SSc mimicking hereditary hemorrhagic telangiectasia $(\mathrm{HHT})$.

\section{CASE REPORT}

M.E.S.C., 54 years old, female, smoker (20 packs/year). Complains of punctate hyperemic lesions in the $2 \mathrm{nd}$, 3rd and 4 th left chirodactyls for 1 year and the appearance of similar lesions in the hard palate for 1 month. She denied constitutional symptoms. History of arthralgia in hands and wrists with morning stiffness of less than 1 hour. The physical examination revealed purple papules on the palmar face of the second, third and fourth left chirodactyls, as well as petechiae on the hard palate. Complete blood count, basic serum chemistry and urinalysis were unremarkable. Hepatitis B and C, HIV, CMV, EBV, parvovirus B19 serologies were negative. Echocardiogram was normal. Palmar skin lesion biopsy was performed. During follow-up, the patient reported an increase in the quantity and size of palmar injuries. Raynaud's phenomenon (RP) and palm telangiectasias (Fig. 1) was seen during the examination. There were no skin thickening, calcinosis or digital ulcers. Lesions persisted on the hard palate (Fig. 2). Erythrocyte sedimentation rate was $50 \mathrm{~mm}$ (1st hour), rheumatoid factor and anti-CCP were negative. Antinuclear antibody (ANA) pattern: centromere 1:640, anticentromere reagent 1: 640, anti-Scl 70 negative. Spirometry showed reduced carbon monoxide diffusion. Chest tomography showed bilateral centrilobular emphysema with mosaic attenuation. Upper digestive endoscopy: antral mild enanthematous gastritis. Esophageal manometry: normal. Nailfold capillaroscopy: dilated capillaries. At skin biopsy, proliferation of capillaries with dilated lumen in the superficial dermis and endothelium without atypia were present. The patient scored 10 at the criteria of ACR/EULAR 2013.

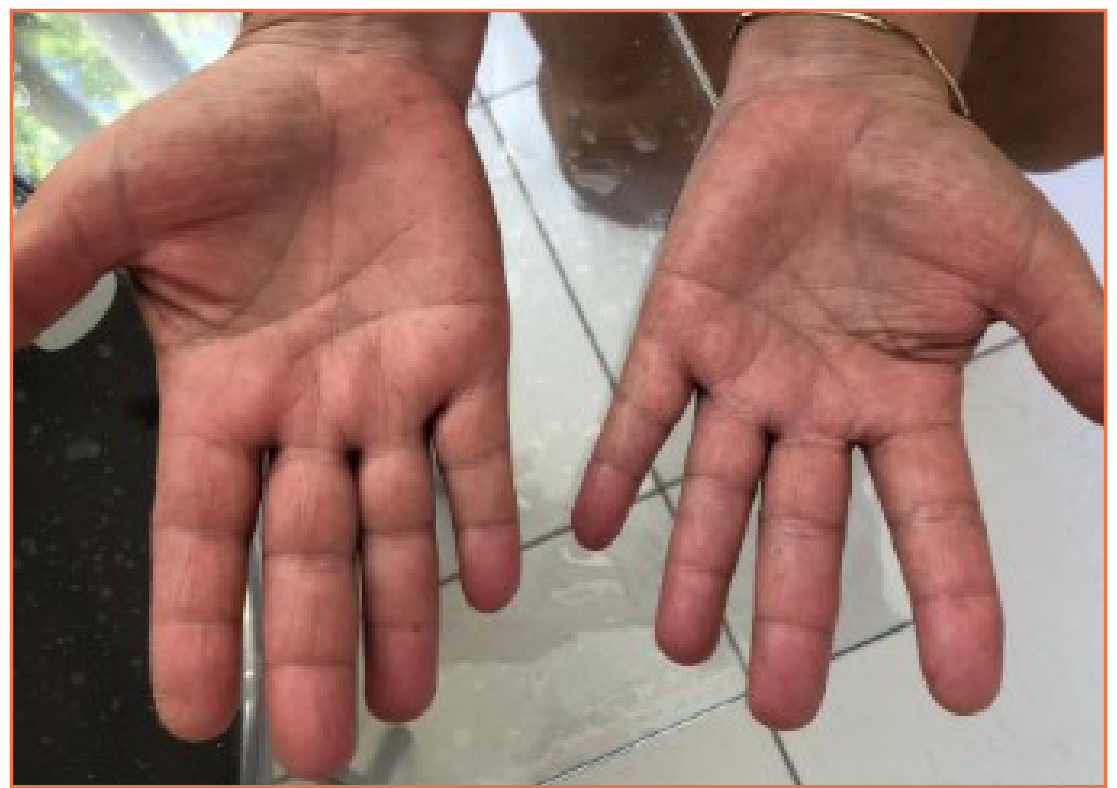

Figure 1. Palmar telangiectasias. 


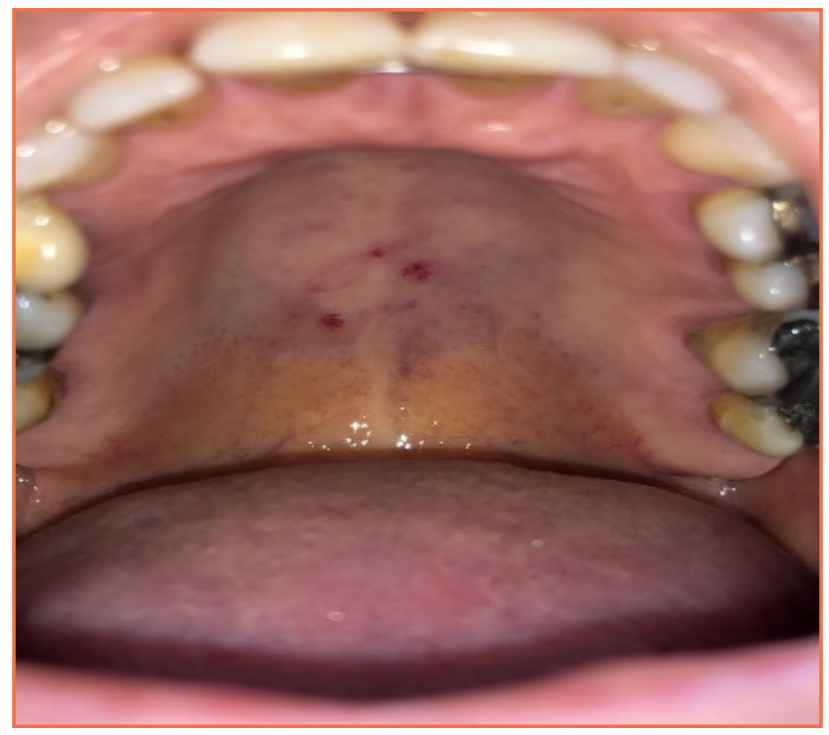

Figure 2. Mucous telangiectasias.

\section{CONCLUSION}

This case highlights the rare initial presentation of SSc with telangiectasia. The EULAR Scleroderma Trial and Research Group has formulated preliminary criteria for the very early diagnosis of SSc. Alert for the diagnosis: RP, finger edema and standard nucleolar centromere ANA pattern. Antinuclear antibody is expected in most patients; its absence requires a differential diagnosis with eosinophilic fasciitis, nephrogenic systemic fibrosis and scleromyxedema. The presence of mucous telangiectasias, in this case, made it necessary to exclude the hypothesis of HHT. Presence of at least 3 of the Curaçao criteria (epistaxis, cutaneous or mucous telangiectasias, visceral involvement and family history) classify this disease. 